\title{
Çimentolu asetabular komponent ve kafes uygulamaları
}

\author{
Cemented acetabular component and cage applications
}

\author{
Sancar Bakırcıoğlu ${ }^{1}$, İbrahim Akel ${ }^{2}$, Bülent Atilla ${ }^{1}$ \\ ${ }^{1}$ Hacettepe Üniversitesi Tıp Fakültesi, Ortopedi ve Travmatoloji Anabilim Dalı, Ankara \\ 2̇zmir Kent Hastanesi, İzmir Ekonomi Üniversitesi, İzmir
}

\begin{abstract}
Çimentolu asetabular komponentler geçtiğimiz yıllarda primer total kalça artroplastisi prosedürlerinde denenmiş olsa da, uzun dönem sağkalımının düşük olması nedeniyle giderek kullanım alanı kıstlanmıştır. Osteoporotik ve yaşam beklentisinin düşük olduğu hastalarda, bazı tümör rekonstrüksiyonlarında ve oldukça fazla miktarda asetabular kemik defektinin olduğu seçili kalça revizyon cerrahisi olgularında tercih edilmektedir. Primer total kalça artroplastisi olgularının artmasına paralel artan revizyon olgularının sayısı da göz önünde bulundurulduğunda; revizyon cerrahisinde iyi bir klinik sonuç elde etmek için uygun bir ameliyat öncesi hasta değerlendirmesi yapmak, çimentolu komponentlerin hangi olgularda, nasıl kullanılabileceğini anlamak ve doğru rekonstrüktif seçeneği uygulamak önemlidir. Tedavi seçenekleri arasında; çimentosuz değişik rekonstrüksiyon metodlarının dışında, çimentolu komponent ile impaksiyon greftleme, halka ve kafes yapıları, kap-kafes rekonstrüksiyonları, oblong cup ve triflange (üç yakalı) rekonstrüksiyon gibi yöntemler bulunmaktadır. Bu yazıda hem primer olarak hem de asetabular revizyonlarda çimentolu komponentlerin kullanılması anlatılacaktır.
\end{abstract}

Anahtar sözcülkler: çimento; asetabulum; revizyon; kalça artroplastisi; kafes; rekonstrüksiyon

\begin{abstract}
Although cemented acetabular components were used in primary total hip arthroplasty procedures in past decades, currently their role decreased because of their low survival rate in long follow-ups. They are preferred in patients with osteoporosis and having short life expectancies, some tumor reconstructions, and selected surgical hip revision cases with large acetabular bone defects. As the primary hip replacement rates increase parallel to revision cases, to achieve a good clinical outcome in revision surgery, it is important to make proper preoperative patient evaluation, to understand in which cases and how the cemented components can be used, and apply the correct reconstructive option. Apart from the uncemented acetabular reconstruction techniques, impaction grafting with cemented component, hook and cage structures, cup-cage reconstructions, oblong cup and triflange reconstruction are the treatment options. In this review, the use of cemented components will be explained both as primary and acetabular revision.
\end{abstract}

Key words: cement; acetabulum; revision; hip arthroplasty; cage; reconstruction
Ç imentolu asetabular komponentin kalça artroplastisindeki yeri kısıtlı olup, çoğunlukla ileri yaşta, kemik kalitesi kötü ve fiziksel aktivitesi çok fazla olmayan, beklentilerin düşük olduğu hastalarda uygulanmaktadır. [1] Çimentolu asetabular tespit bu gibi durumlar dışında; bazı tümör rekonstrüksiyonları ve oldukça fazla miktarda asetabular kemik kaybının olduğu bazı seçili revizyon artroplastisi rekonstrüksiyon seçeneklerinde de tercih edilmektedir. ${ }^{[2]} \mathrm{Bu}$ durumlarda, çimentolu asetabular komponent ile asetabular rekonstrüksiyon halkası sıklıkla birlikte kullanılır. Çok yüksek yoğunluklu kalın polietilenden (UHMWPE) oluşan asetabular komponent, çimento ile kilitlenmeyi kolaylaştırmak için komponentin arkasında yüzeyi arttıran $3 \mathrm{~mm}$ yükseklikte çıkıntılar ve oyuklar içerecek şekilde tasarlanmıştır. Bu tasarım sayesinde, uygun bir çimento dağılımı sağlanarak stabilite arttırılmıştır. Ameliyat sonrası grafilerde, kabın yerinin belirlenmesi, inklinasyon ve anteversiyon açılarının değerlendirilebilmesi için kapların kenarlarına tel yerleştirilmiştir.

- Illetişim adresi: Prof. Dr. Bülent Atilla, Hacettepe Üniversitesi Tıp Fakültesi, 06100 Sıhhiye, Ankara, Turkiye Tel: 0312 - 3051209 e-posta: batilla62@gmail.com

- Geliș tarihi: 24 Ocak 2019 Kabul tarihi:24 Ocak 2019 
Çimentolu asetabular komponent tasarımındaki ve çimentolama tekniklerindeki gelişmeler sonucu; erken dönemde başarılı sonuçlar alınmış olsa da kemik-çimento ara yüzündeki yetersiz biyolojik entegrasyon nedeniyle uzun dönem çalışmalar sonucu radyografik ve klinik gevşeme gözlenmiş ve komponentin uzun dönem sağkalım sonuçları tatmin edici bulunmamıştır. ${ }^{[3]}$ Bu nedenle, günümüzde total kalça artroplastisi (TKA) uygulamaları büyük oranda çimentosuz komponentler tercih edilerek artan hızda devam etmektedir.

\section{TOTAL KALÇA ARTROPLASTISINDE REVIZYON}

TKA'da 10 yıllık başarı oranı \%90'ın üzerinde olmasına rağmen revizyonlar bir yılda uygulanan kalça artroplastisi olgularının \%15'ini oluşturmaktadır. ${ }^{[4]}$ Primer olguların artması, daha genç ve aktif hastalara TKA uygulanması, hastalarda yaşam beklentisinin uzaması, obezite oranının artması gibi faktörler nedeniyle revizyon oranının giderek yükselmesi beklenmektedir. ${ }^{[5]}$

Yaklaşık 50.000 TKA revizyonu uygulanan hastanın dahil edildiği bir çalışmada; revizyon nedeni olarak daha çok mekanik instabilite, aseptik gevşeme sonucu ortaya çıkan osteoliz ve enfeksiyon gibi faktörler belirlenmiş, hastaların \%41'inde hem femoral hem de asetabular komponent, \%13'ünde ise izole asetabular komponent kaynaklı revizyon ihtiyacı duyulduğu belirtilmiştir. ${ }^{[6]}$

Asetabular kemik kayıplarının rekonstrüksiyonu, kalça revizyon artroplastisinde başarıyı belirleyen en önemli etkenlerden biridir. Başarılı bir asetabular rekonstrüksiyon; asetabular implant ile kemik arasında sağlam bir tespit sağlamalı, fizyolojik yükleri çevredeki asetabular kemiğe dağıtan mekanik bir yapı oluşturmalı ve mekanik instabiliteyi önlemek için kalçanın rotasyon merkezini anatomik yerine getirerek normal eklem kinematiğini sağlamalıdır. ${ }^{[7]}$ TKA uygulamalarının ve dolayısı ile revizyon olgularının arttığı bu dönemde, cerrahın ameliyat öncesi planlamayı doğru yapması ve uygun rekonstrüksiyon seçeneklerini anlaması başarılı bir asetabular revizyon cerrahisi için gereklidir.

\section{ASETABULAR KEMIK KAYIPLARININ SINIFLANDIRILMASI}

Asetabular kemik kayıpları için yapılmış sınıflandırma sistemleri, ameliyat içi bulunacak kemik kaybının yeri, şiddeti ve asetabular implantın sağlam tespiti için uygun rekonstrüksiyon seçeneğini olasılıkla belirlemede kullanılır. ${ }^{[8]}$ ilk olarak 1989'da D'Antonio ve ark.'nın yaptıkları The American Academy of Orthopaedic Surgeons (AAOS) sınıflaması, asetabular kemik kayıplarını segmental, kaviter, kombine veya pelvik devamsızlık ve kalça artrodezi olarak ayırmaktadır. ${ }^{[9]}$

Kaviter kemik kayıpları asetabular kavite içerisinde lokalize olur ve anterior, superior ve posterior kenarı sağlamken asetabular çatıda kemik kaybı olan lezyonları içerir. Segmenter ve kaviter kemik kayıpları ayrı ayrı görülebileceği gibi bir arada da görülebilir ve kemik kaybının yerleşimine göre; anterior, superior, posterior, periferal veya santral olmak üzere alt gruplara ayrilır. Bu sınıflama sistemi, basit ve pratik olmasına rağmen kemik kaybının yeri ve büyüklüğü hakkında kantitatif bilgi vermediği için günümüzde çok fazla kullanılmasa da, halen en çok atıf alan çalışmadır (Tablo 1).

Engh, Gross ve Saleh gibi birçok sınıflandırma sistemi bulunsa da, bunların arasında en sık kullanılan Paprosky'nin asetabular kemik kayıplarını sınıflama sistemidir. ${ }^{[10]}$ Kemik kaybının tipi, boyutu ve lokalizasyonu hakkında daha iyi bilgi verdiği ve revizyon cerrahisi için uygun rekonstrüktif alternatifleri seçmede yardımcı olduğu, çalışmalarla gösterilmiştir. ${ }^{[11]} \mathrm{Bu}$ sınıflamanın amacı, revizyon sırasında çimentosuz hemisferik komponentlerin kullanılmasına izin verecek kemik yapı varlığını ameliyat öncesi değerlendirmektir. İçeriğinde dört değişken bulunur. Bunlar; rotasyon merkezinin yer değiştirmesi, iskial osteoliz miktarı, kemik kaybının Kohler hattına (ilioiskial hat) ulaşıp ulaşmadığı ve gözyaşı bölgesinin bozulma derecesidir. Bu dört değişkenin sınıflanmada kullanılması; superior

Tablo 1. Asetabular kemik kayıplarında AAOS sınıflaması ${ }^{[9]}$

\begin{tabular}{cc}
\hline Tip I & Segmental kemik kayıpları \\
Periferal & Superior \\
& Anterior \\
& Posterior \\
& Santral (mediyal duvar kayıpları) \\
Tip II & Kaviter kemik kayıpları \\
& Periferal \\
& Superior \\
& Anterior \\
& Posterior \\
Tip III & Santral (mediyal duvar sağlam) \\
Tip IV & Pelvik devamsılı kay \\
Tip V & Artodez
\end{tabular}


çatı, mediyal asetabular duvar ve posterior kolondaki kemik kaybının objektif olarak değerlendirilmesini sağlar (Tablo 2).

\section{ÇiMENTOLAMA TEKNIĞi VE ASETABULAR REVIZYONDA REKONSTRÜKSIYON SEÇENEKLERI}

Asetabular komponent yetmezliği ve bunla ilişkili kemik kayıplarının tedavisi; hastanın özelliklerine, kemik kaybının şiddeti ve yerleşim yerine, biyolojik tespiti destekleyecek sağlam kolonların varlığına ve pelvik devamsızlığın olup olmamasına bağlı olarak değişebilir. Asetabular revizyon rekonstrüksiyonlarının temel amacı, stabil bir tespit sağlamak ve kalçanın rotasyon merkezini normal konumuna getirmektir. Uzun dönem sağkalımın yüksek olması için, doğru rekonstrüksiyon seçeneğini uygulamak ve çimentolama tekniğini iyi bilmek oldukça önemlidir.

\section{Çimentolama Tekniği}

Asetabulum cerrahi olarak ortaya çıkarıldıktan sonra, anterior ve posterior kolonda kansellöz kemiğe ulaşılana kadar, kademeli oyma işlemi uygulanır. Konak kemik stoğuna zarar vermemek için aşırı mediyalizasyona dikkat edilmelidir. Çimento ile olan entegrasyonu arttırmak için ilium ve iskiumun subkondral kemiklerine uzanan, $6 \mathrm{~mm}$ 'lik tespit delikleri açılır. Mediyal duvara delik açmak, uygulanan çimentonun pelvise düşme riskini taşıdığından dolayı önerilmez. Asetabulum ve açılan deliklerin içindeki serbest kemik parçaları pulsatil yıkama ile temizlendikten sonra, adrenalin emdirilmiş gazlı bezler ile hemostaz sağlanır. Takiben, çimento kansellöz kemik üzerine önce açılan deliklerden başlayarak basınçlı uygulama yoluyla yerleştirilir, özellikle "gözyaşı" figürünün altındaki fazla çimento, komponentin öncelikle yerleştiği yer olması nedeniyle, dikkatlice temizlenir. Polietilen kap, tutucusu ile birlikte öncelikle inferiora ve daha sonra mediyal ve superior yüzeye bastırılarak yerleştirilir. Kap, mediyale basınç uygulanarak uygun pozisyonda dondurulur ve bu arada, etraftaki fazladan çimento artıkları mutlaka temizlenmelidir. Çimento tamamen sertleştikten sonra yerleştirilen komponentin stabilitesini, farklı bölgelerine bir impaktör yardımı ile bastırarak, test etmek gerekir. Herhangi bir hareket algılanırsa veya ara yüzden kan veya küçük kabarcıklar çıkarsa, komponent gevşek demektir ve çıkarılıp değiştirilmesi gerekir. İmplantın kenar kısmında çıkıntı yapan herhangi bir osteofiti veya çimentoyu mutlaka çıkarmak gerekir, çünkü bunlar impingement ve ameliyat sonrası dislokasyona neden olabilir. Çimentolu asetabular komponentlerin uzun dönem sağkalımları erken ameliyat sonrası dönemde çekilen radyografilerdeki radyolüsen alanlarla ilişkili olduğundan dolayı, çimentonun kansellöz kemiğe penetrasyonunu arttırmak için kuru bir zemin hazırlamak ve çimentolama tekniğini iyi bilmek oldukça önemlidir (Şekil 1).

\section{İmpaksiyon Greftleme}

Asetabular impaksiyon greftleme; sementli bir polietilen kap ile iyice impakte edilmiş kansellöz kemik greftleri aracılığıla kemik stoğunu yeniden sağlamayı amaçlar. Basit kaviter kemik kayıplarında da tek başına bu teknik kullanışı olabileceği gibi, segmenter veya kombine kayıplarda metal mesh'lerle birlikte kullanımı da uygundur. İmpaksiyon greftleme tekniği de, giderek yerini gevşeme oranı daha düşük olan güncel tekniklere bırakmaktadır. Busch ve ark., segmenter, kaviter veya kombine kemik kaybı olan ortalama 50 yaşından genç 42 hastalık bir kohort grubunda, çimentolu polietilen liner ile impaksiyon greftlemeyi takiben uzun dönem sonuçlarını yayımlamış ve 20 yıllık sağkalımın \%82, 25 yıllık sağkalımın ise \%77 olduğunu vurgulamışlardır. ${ }^{[12]}$ Atilla ve ark.'nın 1996-2003 yılları arasında 39 hastanın 40 kalçasında uyguladıkları revizyon

Tablo 2. Asetabular kemik kayıplarında Paprosky sınıflaması ${ }^{[10]}$

\begin{tabular}{llccc}
\hline Tip & Rotasyon merkezi migrasyonu & İskial osteoliz & Kohler hattı & Gözyaşı \\
\hline I & Yok & Yok & Sağlam & Sağlam \\
IIA & Zayıf $(<3 \mathrm{~cm})$ & Yok & Sağlam & Sağlam \\
IIB & Orta $(<3 \mathrm{~cm})$ & Zayıf & Sağlam & Sağlam \\
IIC & Zayıf $(<3 \mathrm{~cm})$ & Zayıf & Bozulmuş & Orta derece lizis \\
IIIA & Şiddetli $(>3 \mathrm{~cm})$ & Orta & Sağlam & Orta derece lizis \\
IIIB & Şiddetli $(>3 \mathrm{~cm})$ & Şiddetli & Sozulmuş & Şiddetli lizis
\end{tabular}




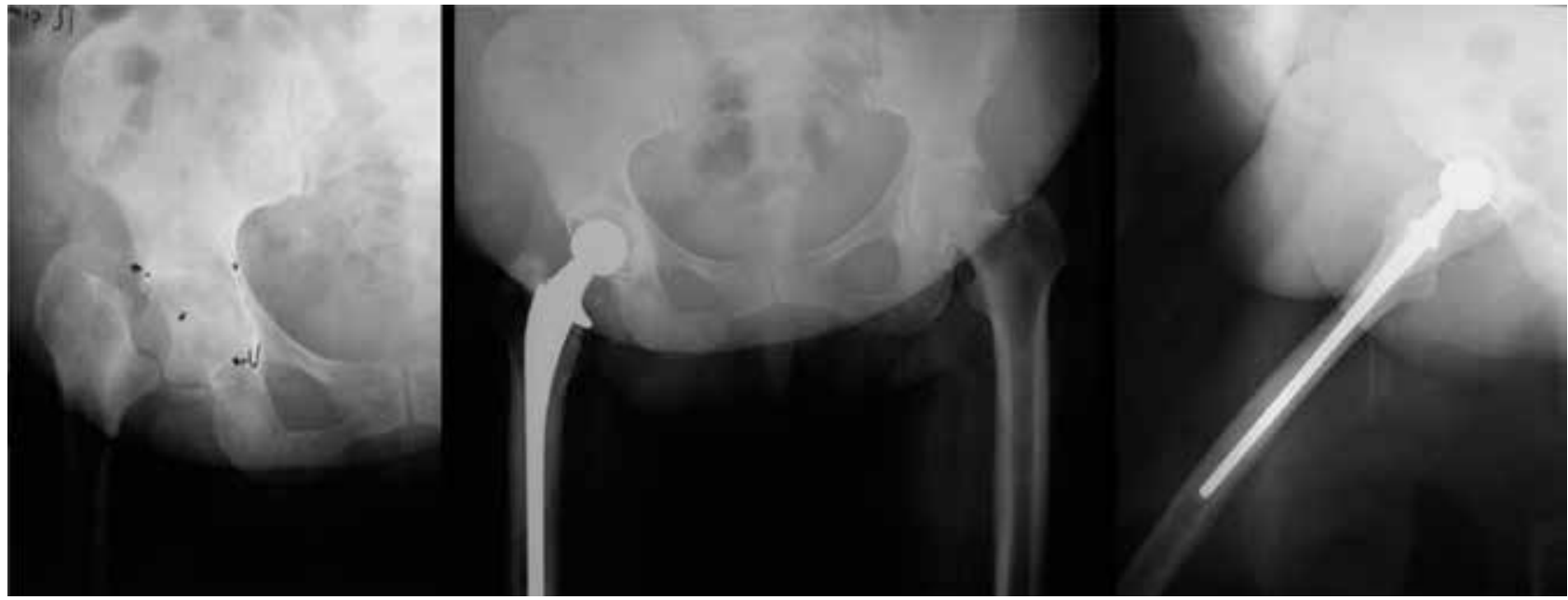

Şekil 1. Meme kanseri nedeniyle patolojik femur boyun kırığı olan 60 yaşındaki kadın hastaya uyguladığımız çimentolu TKA.

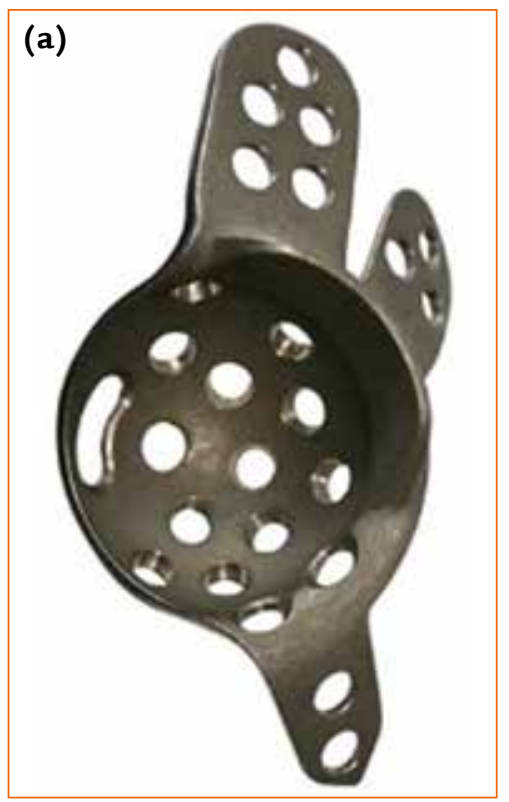

(b)

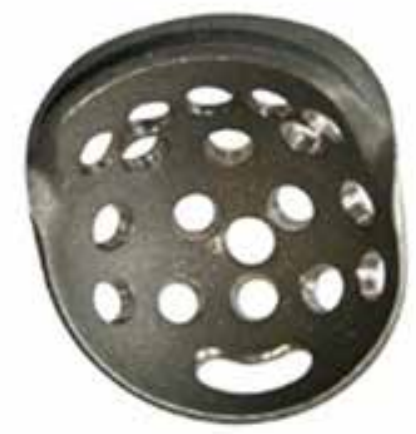

Şekil 2. a, b. Kanatlı asetabular kafes; büyük kaviter defektler, mediyal duvar defektleri, anterior veya posterior duvarı içeren segmental defektler ve pelvik devamsızlık gibi ciddi kemik kayıpları durumunda tercih edilir (a). Çatı takviye halkası konak kemiğine karşı supero-posterior ve infero-mediyal olarak yeterli destek sağlamak için kullanılır (b). Endikasyonları arasında ciddi osteoporoz, asetabular erozyon, multipl kistler, hafif protrüzyon ve kaviter kusurlar vardır. artroplastisinde; ortalama beş yıllık takip süresi sonucunda impaksiyon greftleme ile revizyon uygulanan 15 hastada \%26,3 oranında aseptik gevşeme izlenirken, kafes tekniği uygulanan 25 hastada bu oran \%8,3 idi. $\mathrm{Bu}$ sonuç ayrıntılı incelendiğinde, impaksiyon greftleme yapılan hastalardan özellikle segmenter defekti olanlarda başarının düşük olması; konulan greftlerin yeterince stabil olmamasına ve bu nedenle bu grup hastalarda greft-konak konsolidasyon eksikliğine bağlandı. O tarihten sonra, Hacettepe uygulamasında segmenter defektli hastalarda greft mutlaka üzerine konulan bir kafes yardımıyla korunarak rekonstrüksiyon yapılmaktadır.

\section{Halka ve Kafes Rekonstrüksiyonu}

Greft osteo-entegasyonunu sağladıkları ve kemik stoğunu arttırmada yardımcı oldukları için, ciddi kemik kayıpları durumunda halka ve kafesler de rekonstrüksiyon seçenekleri olarak düşünülebilir. Bunlar, kemiğin remodelling fazında yapısal veya morselize allogrefti korumak için scaffold (iskele) görevi görür. Kullanılan iki tip halka; çatı takviye halkası (roof reinforcement ring) ve anti-protrüzyo kafesidir (Şekil 2). Çatı takviye halkası asetabular çatıyı, anti-protrüzyo kafesler ise iliumdan iskiuma uzanarak bütün asetabulumu korur. Çatı takviye halkaları, en iyi sonuçları kaviter ve anterior segment defektlerinde vermektedir. Bunlar, 
asetabular zemin veya kaviter defektlerinin rekonstrüksiyonu ve asetabular kenarın lateral sınırındaki daha küçük kemik defektleri için tasarlanmıştır; ayrıca, rotasyon merkezinin anatomik pozisyonunu tekrar yerine getirmeye de izin verir. Halka yapısının kancasını incisura asetabula'ya sabitleyebilmek için, asetabulumun inferior kısmı sağlam olmalıdır. Anti-protrüzyo kafesler, pelvik devamsızlık gibi daha ciddi kemik kayıplarının olduğu durumlarda endikedir. Vidaların iyi kemik stoğu kalan yerlere yerleştirilmesi gerektiğinden dolayı, kullanımları iyi bir cerrahi yaklaşım ve tecrübe gerektirir. Halkaların ve kafeslerin başlıca avantajları; kafes ve halkanın kalça merkezinin restorasyonuna izin vermeleri, allogrefte uniform yüklenme sağlayarak kemik yeniden şekillenmesini uyarmaları, greftin konak kemiğine birleşmesini arttırmaları ve cerrahın liner'ı halkadan bağımsız olarak istediği pozisyonda çimentolamasına olanak sağlamalarıdır. Kafes yapısının diğer avantajları; morsalize ya da yapısal allogrefti koruması ve kafesin başarısız olması durumunda çimentosuz revizyon yapılabilmesidir. ${ }^{[13]}$ Kafesin kırılması veya gevşeme riski ise biyolojik tespit eksikliğinden ötürü meydana gelebilen en büyük dezavantajıdır. İmpaksiyon greftleme üzerine anti-protrüzyo kafesleri kullanılan olgularda, kafes ve kalan konak kemiği arasında mutlaka geniş bir temas alanı olmalı ve en az 3-4 vida ile rijid primer tespit uygulanmalıdır.

\section{Çatı halkasının yerleştirilmesi}

Kemik stoğunun restorasyonundan sonra, kemik yatağın çapı çatı halkasının dış çapına uygun olmalıdır. Çatı halkası yerleştirilirken yakalığı superiora ve hafifçe posteriora yerleştirilir. Halkanın konak kemiğine karşı superior, posterior ve infero-mediyal kısımda stabil olduğundan emin olunmalıdır. Vidalar asetabular kubbeye doğru yük binen superior kesime yerleştirilir. Hastanın kemik kalitesi iyi ise periferal vidalarda yerleştirilebilir, ancak büyük damarlara yakınlığı nedeniyle anterior ve mediyale doğru vida yerleştirilmekten kaçınılmalıdır. Superior yakalık ve çatı halkasının inferior kısmı kemik greftinin üzerine oturtulmamalıdır. Aksi halde halka stabilitesi sağlanamaz ve yerleştirilen vidalar kırılabilir. Bu durumda, asetabular kubbeye en az üç vida ile yerleştirilecek şekilde bir kafes daha doğru bir seçenek olabilir.

\section{Kafesin yerleştirilmesi}

Kafes, iliumdan iskiuma uzanan geniş bir cerrahi yaklaşım gerektirir. Geleneksel transvers trokanterik osteotomi gerekli olabilir. Defekti iyi bir şekilde tanımlamak için asetabulumun tüm çevresini yeterince açığa çıkarmak önemlidir. Kemik stoğunun restorasyonundan sonra, kemik yatağın çapı kafesin dış çapına uygun olmalıdır. Kafesin superior yakası iliumun superioruna ve hafifçe arkaya doğru yerleştirilerek stabilize edilir. İnferior yakası ise iskiumun üstüne yerleştirilir. Asetabular kubbeye bir veya iki vida yerleştirildikten sonra iliak kanat ve iskiuma yerleştirilmiş olan superior ve inferior yakalara iki veya üç adet vida yerleştirilir. Bazı defekt tiplerinde, inferior yakaya vida konulmaksızın destek etkisi elde edilmesi beklenir. Kliniğimizde uyguladığımız teknikte, inferior kanadın iskial kemiğin medüller kanalına yerleştirilmesi kritiktir. Bu, iskial kemiğin korteksleri arasında stabil ve kalıcı bir tespit ve eklemin rotasyon merkezini anatomik yerine getirerek, normal eklem kinematiğini sağlamaktadır. Ek vida tespiti olmaksızın iskial kanadın iskial kemiğe gömülmesi, iskium korteksleri arasında stabilce kalıcı bir tespit sağlar. Bu şekilde, kafes yeterli derecede mediyalize edilerek eklemin rotasyon merkezi anatomik yerine getirilir ve normal eklem kinematiği sağlanır. Sonraki etapta, önce santral daha sonra üst kanattaki vidaların yerleştirilmesi ile stabilizasyon güçlendirilir. Kafesin santral kısmına yerleştirilen vidalar sayesinde ise impakte allogreftlerin kafes ile kompresyonu yoluyla santralizasyonlarının devamı sağlanmaktadır. (Şekil 3). Segmental defektler ya da pelvik devamsızlıkta ise inferior yakadan iskiuma doğru vida yerleştirilirken, siyatik sinir mutlaka bulunarak koruma altına alınmalıdır.

Halka ve kafes kullanımının orta dönem sonuçları tatmin edicidir. Zehtner ve Ganz'ın yaptıkları, 27 hastanın ortalama 7,2 yıl takip edildiği bir çalışmada, çatı destekleyici halkanın 10 yıllık sağkalımının $\% 80$ olduğu belirtilmiştir. ${ }^{[14]}$ Goodman ve ark.'nın ortalama 4,6 yıllık takip süresiyle takip ettiği 42 hastalık bir çalışmada, Burch-Schneider kafeslerinin sağkalımının $\% 76$ olduğu görülmüştür. ${ }^{[15]}$ Atilla ve ark.'nın, 67 hastanın 71 kalçasında uyguladıkları partiküler kemik grefti ve kafes tekniğinde, 17 yıla varan takip süresi içinde dört hastada aseptik gevşeme görülürken, protezin sağkalımı iki yılda \%94,6, beş yılda \%91,6, toplamda ise \%91,9, ortalama revizyonsuz süre 15,8 yıl olarak bulunmuştur. Uzun dönem sonuçları tatmin edici olsa da, hem gevşeme ve kırılma riskinden dolayı hem de modüler poroz metal augmentlerin kullanımının artması nedeniyle, halka ve kafes kullanımı, geniş kemik kaybı olan ve çok uzun süreli tespit gerekmeyen yaşlı hasta grubunda seçilmelidir.

\section{Kap-Kafes Rekonstrüksiyonu}

Yeni tekniklerden biri olan kap-kafes rekonstrüksiyonu; biyolojik olarak sabitlenmiş bir poroz metal kap üzerine yerleştilmiş bir ilioiskial kafesten oluşur (Şekil 4). Sadece Paprosky Tip IIIA ve IIIB kemik kayıplarında değil, ayrıca pelvik devamsızlıklarda da kullanılır. Bu teknikte, çimentosuz asetabular 




Şekil 3. a, b. Otuz beş yaşında kadın hasta; sağ kalça eklemine uygulanmış çimentolu protezde aseptik gevşeme (a); asetabular kafes ve kemik grefti ile beş yıllık başarılı sonuç (b).



Şekil 4. Ciddi pelvik devamsızlığı olan 48 yaşındaki kadın hastada uyguladı̆̆ımız kap-kafes yöntemi ile başarılı TKA revizyonu. komponent tek başına ya da poroz metal augment ile konakçı kemiğine sabitlenir; daha sonra anti-protrüzyo kafes, pelvik devamsızlığı sabitlemek için çimentosuz komponentin üzerine yerleştirilir. illium ve iskiuma yerleştirilen vidalar sayesinde, kafes pelvik devamsızlığın ilk stabilizasyonunu sağlar ve böylece çimentosuz komponent ya da metal desteklerin kemiğe biyolojik olarak sabitlenmesi için gereken zaman yaratılmış olur. Alfaro ve Fernández, pelvik devamsızlığı olan beş hastada trabeküler metal buttress augmentleri ile kombine edilmiş "kap-kafes" yapının kullanımını tarif etmişlerdir ve ortalama 26 aylık takipte hiçbir hastada başarısızlık görülmemiş̧tir. ${ }^{[16]}$

\section{Triflange (Üç Yakalı) Rekonstrüksiyon}

Genellikle Paprosky Tip IIIA ve IIIB defektlerde kullanılan, hasta pelvisi üç boyutlu bilgisayarlı tomografi yardımı ile değerlendirilerek yapılan, hastaya özel üretilmiş poroz veya hidroksiapatit kaplı cihazlardır. ${ }^{[17]}$ Bu kişiye özel üretilmiş yapılar, yapının ilium, iskium ve pubise biyolojik olarak sabitlenmesini sağlar. Pelvik devamsızlıklarda anti-protrüzyo kafesleri ve halkaların aksine triflange (üç yakalı) komponenti rijid bir tespit sağlar ve kemik iyileşmesi üzerine etkisi daha çoktur. [18] Dennis ve ark., triflange komponentle revize edilen 24 kalçada dört yıllık takip süresi boyunca sadece bir olguda aseptik gevşeme görmüşlerdir. ${ }^{[19]}$ Her ne kadar 
orta-uzun dönem sonuçları iyi olsa da, triflange yapı ile ilgili kaygılar; toplam maliyeti, biyolojik ingrowth gerçekleşip gerçekleşmeyeceği ve sert metal yapının konakçı kemiği üzerindeki uzun süreli etkisini içerir.

\section{SONUÇ}

Primer olgularda çimentolu asetabular komponent kullanımı büyük çoğunlukla terk edilmiştir; ancak yine de, başarılı bir asetabular revizyon cerrahisi için, çimentolama tekniklerine de hakim olup çimentolu rekonstrüksiyon ve buna uygun ameliyat öncesi planlama ve cerrahi yönetim seçeneklerini iyi bilmek gerekir. Kemik kaybı miktarının cerrahi öncesinde belirlenmesi oldukça önemlidir. İyi bir revizyonda hedef, yetersiz implantların konak dokusu ve kemiğine minimal hasar verilerek çıkarılması ve sonrasında hastanın fonksiyonunu arttırıp ağrılarını giderecek implantların, kemik defektlerinin restorasyonu ile birlikte konak kemiğine yerleştirilmesidir. Çoğu olguda tedavi hemisferik kap ve destekleyici vidalar ile uygulanabilse de, kemik kaybının fazla olduğu durumlarda çimentolama tekniklerinin de uygulanmasını gerektiren, halka ve kafes yapıları ile birlikte, yapısal ya da partiküler kemik greftleri, kap-kafes yapıları veya bireyselleştirilmiş triflange komponentler de tedavide kullanılabilir. Giderek artan revizyon olgu sayısı göz önünde bulundurulduğunda, günümüzde kullandığımız mevcut biyo-materyal ve çimentolu rekonstrüksiyon seçeneklerini de içeren implantların uygun cerrahi seçenekler olduğunun daha geniş kapsamlı klinik çalışmalarla gösterilmesi ve yeni geliştirilecek rekonstrüksiyon seçeneklerine yönelik araştırmalar yapılması gerekmektedir.

\section{KAYNAKLAR}

1. Clement ND, Biant LC, Breusch SJ. Total hip arthroplasty: to cement or not to cement the acetabular socket? A critical review of the literature. Arch Orthop Trauma Surg 2012;132(3):411-27. Crossref

2. Taylor ED, Browne JA. Reconstruction options for acetabular revision. World J Orthop 2012;3(7):95-100. Crossref

3. Mulroy RD, Harris WH. The effect of improved cementing techniques on component loosening in total hip replacement. An 11-year radiographic review. J Bone Joint Surg Br 1990;72$\mathrm{B}(5): 757-60$. Crossref

4. Furnes O, Lie SA, Espehaug B, Vollset SE, Engesaeter LB, Havelin LI. Hip disease and the prognosis of total hip replacements: a review of 53.698 primary total hip replacements reported to the Norwegian arthroplasty register 1987-99. J Bone Joint Surg Br 2001;83-B(4):579. Crossref
5. Kurtz SM, Ong KL, Lau E, Bozic KJ. Impact of the economic downturn on total joint replacement demand in the United States: updated projections to 2021. J Bone Joint Surg Am 2014;96(8):624-30. Crossref

6. Bozic KJ, Kurtz SM, Lau E, Ong K, Vail TP, Berry DJ. The epidemiology of revision total hip arthroplasty in the United States. J Bone Joint Surg Am 2009;91(1):128-33. Crossref

7. Haynes JA, Stambough JB, Sassoon AA, Johnson SR, Clohisy JC, Nunley RM. Contemporary surgical indications and referral trends in revision total hip arthroplasty: a 10-year review. J Arthroplasty 2016;31(3):622-5. Crossref

8. Sporer SM. How to do a revision total hip arthroplasty: revision of the acetabulum. J Bone Joint Surg Am 2011;93(14):135964. Crossref

9. D'Antonio JA, Capello WN, Borden LS, Bargar WL, Bierbaum BF, Boettcher WG, Steinberg ME, Stulberg SD, Wedge $\mathrm{JH}$. Classification and management of acetabular abnormalities in total hip arthroplasty. Clin Orthop Relat Res 1989;(243):126-37. Crossref

10. Paprosky WG, Perona PG, Lawrence JM. Acetabular defect classification and surgical reconstruction in revision arthroplasty: a 6-year follow-up evaluation. J Arthroplasty 1994;9(1):33-44. Crossref

11. Saleh KJ, Holtzman J, Gafni A, Saleh L, Jaroszynski G, Wong P, Woodgate I, Davis A, Gross AE. Development, test reliability and validation of a classification for revision hip arthroplasty. J Orthop Res 2001;19(1):50-6. Crossref

12. Busch VJJF, Gardeniers JWM, Verdonschot N, Slooff TJJH, Schreurs BW. Acetabular reconstruction with impaction bone-grafting and a cemented cup in patients younger than fifty years old: a concise follow-up, at twenty to twentyeight years, of a previous report. J Bone Joint Surg Am 2011;93(4):367-71. Crossref

13. Gross $A E$, Goodman $S$. The current role of structural grafts and cages in revision arthroplasty of the hip. Clin Orthop Relat Res 2004;429:193-200. Crossref

14. Zehntner MK, Ganz R. Midterm results (5.5-10 years) of acetabular allograft reconstruction with the acetabular reinforcement ring during total hip revision. J Arthroplasty 1994;9(5):469-79. Crossref

15. Goodman $S$, Saastamoinen $H$, Shasha $N$, Gross $A$. Complications of ilioischial reconstruction rings in revision total hip arthroplasty. J Arthroplasty 2004;19(4):436-46. Crossref

16. Alfaro JJB, Fernández JS. Trabecular Metal buttress augment and the Trabecular Metal cup-cage construct in revision hip arthroplasty for severe acetabular bone loss and pelvic discontinuity. Hip Int 2010;20(7 Suppl):119-27. Crossref

17. Colen S, Harake R, De Haan J, Mulier M. A modified custommade triflanged acetabular reconstruction ring (MCTARR) for revision hip arthroplasty with severe acetabular defects. Acta Orthop Belg 2013;79(1):71-5.

18. Myncke I, van Schaik D, Scheerlinck T. Custom-made triflanged acetabular components in the treatment of major acetabular defects. Short-term results and clinical experience. Acta Orthop Belg 2017;88(3):341-50.

19. Dennis DA. Management of massive acetabular defects in revision total hip arthroplasty. J Arthroplasty 2003;18(3):1215. Crossref 